Objectives The purpose of this study was to assess the prevalence of WPB among anaesthesiology caregivers and to identify its impact on their mental health.

Methods A cross-sectional study was conducted over three months among the anaesthesiology caregivers of the three university hospitals of Mahdia and Monastir in Tunisia $(n=129)$. A self-administrated survey was used, including the standardized Negative Acts Questionnaire-Revised (NAQ-R) to evaluate the WPB. The impact on mental health was assessed by the Rosenberg self-esteem scale, the Beck Depressive Inventory and the Hamilton Anxiety Rating Scale.

Results The response rate was $55 \%$. The sex-ratio (M/W) was 0.57 and the mean age was $36.1 \pm 1.1$ years. Among the respondents, the prevalence of bullying, measured by the NAQ-R score, was $36.5 \%$. Moreover, $46.7 \%$ of anaesthesiology caregivers self-labelled themselves as victims of WPB. According to the Beck Depressive Inventory, $61.7 \%$ of the anaesthesiology staff were depressed. Anxiety disorders were noted in $49.9 \%$ of cases. The Self-esteem was 'very low' to 'low' among $40 \%$ of the caregivers. Multivariate analysis showed that WPB was significantly associated with female gender $(p=0.009)$, the status of medical residents $(p=0.021)$, atypical working hours $(\mathrm{p}=0.008)$, lower self-esteem $(p=0.002)$, and anxiety $(p=0.004)$. No correlation was found between WPB and depression.

Conclusion The mental health of caregivers, especially in intensive care units, determines the quality of care and the patient safety. This mental health is negatively impacted by WPB. Thus, the worrisome high rates of WPB observed in the intensive-care and anaesthesiology environment require the implementation of preventive measures in order to overcome this behavior.

\section{P-396 SOCIAL SECURITY BENEFITS GRANTED TO BRAZILIAN WORKERS WITH OCCUPATIONAL CANCER}

${ }^{1}$ Thamyres Morgado de Almeida, Cristiane Helena Gallasch, Magda Faria, Ricardo Rafael, João Silvestre Silva-Junior. ${ }^{~ U n i v e r s i d a d e ~ D o ~ E s t a d o ~ D o ~ R i o ~ D e ~ J a n e i r o, ~ B r a z i l ~}$

\subsection{6/OEM-2021-EPI.310}

Introduction Actions to control cancer in Brazil were introduced in the early 20th century, conducted with a greater focus on diagnosis and treatment. The emphasis on prevention was limited by the lack of knowledge about the etiology of the disease. Occupational cancer originates from exposure to carcinogenic agents present in the workplace, even after exposure is suspended. It is common for a long period of exposure to elements or risky conditions in the workplace to occur, which makes difficult to diagnose certain types of cancer.

Objective To investigate the variation in the occurrence of occupational cancer among Brazilian workers.

Methods We conducted an ecological study with an investigation of temporal trends, in a quantitative approach, with exploration of absolute and relative frequencies of data on all Brazilian workers who were affected by occupational cancer and who were beneficiaries of the social system.

Results From 2006 to 2012, 798,885 social security benefits were granted to Brazilian workers due to cancer, with an average of 1092,6 cases/year $(S D=502,9)$ of occupational cancer, and average occurrence rate of $0.62 \%$ among all cases of cancer. Considering only occupational neoplasms, there was a higher occurrence of melanomas and other skin neoplasms (n $=2141,55.99 \%)$, neoplasms of the respiratory system $(\mathrm{n}=$ 493, 12.89\%) and lymphatic and hematological tissue neoplasms ( $n=447,11.69 \%$ ).

Conclusion Although preventable, occupational cancer is recurrent among Brazilian workers. The main strategy for reducing risks is to reduce or eliminate exposure to carcinogenic agents within the work environment. The role of nurses in the work environment has become essential, since it contributes to a significant drop in the rate of accidents and occupational diseases, promoting quality of life, identifying risks and implementing health protective measures.

\section{P-397 OCCUPATIONAL DERMATOSES IN PARACHUTE RIGGERS AT A PARATROOPER TRAINING SCHOOL}

${ }^{1}$ VK Sashindran, Rahul Singh. ${ }^{1} 7$ Air Force Hospital, India

\subsection{6/OEM-2021-EPI.311}

Introduction Parachute riggers are specialised tradesmen involved in packing parachutes. Occupational dermatoses has never been studied in them despite their job entailing hard manual work.

Objective To observe and record hand and nail changes in parachute riggers.

Methods Parachute riggers working at the Paratrooper Training School (PTS) Agra consenting to participate in the study were enrolled. A brief history and hand and nail changes seen using a hand-lens were recorded. Data was analysed using R Project for Statistical Computing version R 3.6.2 for Windows.

Results Seventy-six parachute riggers were examined. All had some change and many had more than one change. The mean age was 29.66 years and mean duration of employment in PTS was 29.73 months. All were males. Callosities and loss of cuticle were the commonest abnormalities seen and were present in $61(80.26 \%)$ and $57(75 \%)$ subjects, respectively. Other common changes noted were fissures, xeroderma, pigmentation and dermatitis. Onychomycosis, onychoschizia and splinter haemorrhages were the commonest nail changes seen. Multiple correspondence analysis showed that there was a clustering of all findings barring deformities, digital resorption, paronychia and xeroderma. Odds for chronic irritant dermatitis was related to age of worker and duration of employment. This study is the first study documenting occupational dermatoses in parachute riggers. Since most of them did not use protective gear, repeated trauma due to thorns, and seeds ensnared in parachute fabric, micro-trauma resulting from friction and constant dryness were factors predisposing them to irritant contact dermatitis. Constant pressure during packing predisposed them to splitting of nails, and splinter haemorrhages.

Conclusion Riggers are special tradesmen involved in all steps of parachute packing. All riggers had some manifestation of occupational skin disease. Most of the changes observed were secondary to repeated friction and pressure insults and dryness of the hands. 